Prepared for the U.S. Department of Energy

under Contract DE-AC05-76RL01830

\title{
Ultra High-Rate Germanium (UHRGe) Modeling Status Report
}

\author{
GA Warren \\ DC Rodriguez
}

June 2012

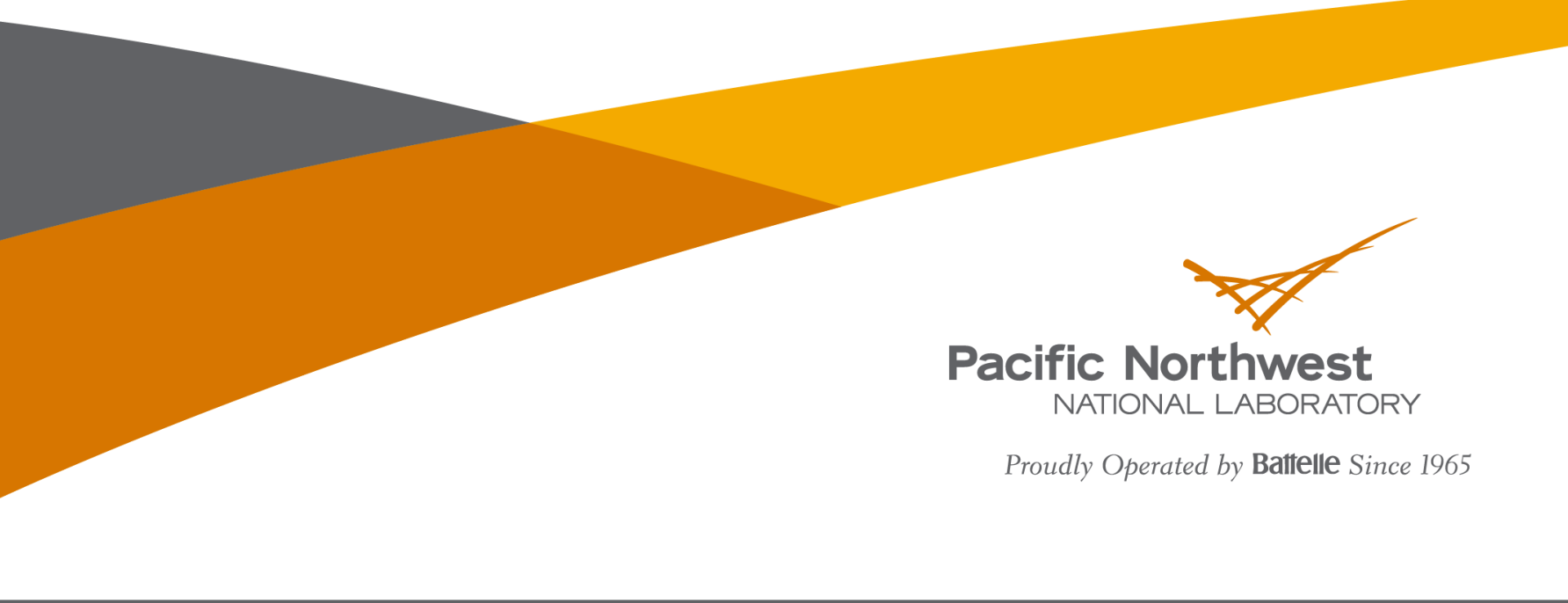




\title{
DISCLAIMER
}

This report was prepared as an account of work sponsored by an agency of the United States Government. Neither the United States Government nor any agency thereof, nor Battelle Memorial Institute, nor any of their employees, makes any warranty, express or implied, or assumes any legal liability or responsibility for the accuracy, completeness, or usefulness of any information, apparatus, product, or process disclosed, or represents that its use would not infringe privately owned rights. Reference herein to any specific commercial product, process, or service by trade name, trademark, manufacturer, or otherwise does not necessarily constitute or imply its endorsement, recommendation, or favoring by the United States Government or any agency thereof, or Battelle Memorial Institute. The views and opinions of authors expressed herein do not necessarily state or reflect those of the United States Government or any agency thereof.

\author{
PACIFIC NORTHWEST NATIONAL LABORATORY \\ operated by \\ BATTELLE \\ for the \\ UNITED STATES DEPARTMENT OF ENERGY \\ under Contract DE-AC05-76RL01830
}

Printed in the United States of America
Available to DOE and DOE contractors from the Office of Scientific and Technical Information,
P.O. Box 62, Oak Ridge, TN 37831-0062;
ph: (865) 576-8401
fax: $(865)$ 576-5728
email: reports@adonis.osti.gov

\begin{abstract}
Available to the public from the National Technical Information Service, U.S. Department of Commerce, 5285 Port Royal Rd., Springfield, VA 22161 ph: (800) 553-6847 fax: $(703) 605-6900$ email: orders@ntis.fedworld.gov online ordering: http://www.ntis.gov/ordering.htm
\end{abstract}

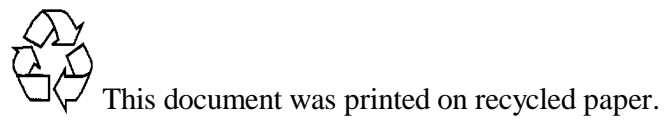




\title{
Ultra-High Rate Germanium (UHRGe) Modeling Status Report
}

\author{
GA Warren \\ DC Rodriguez
}

June 2012

Prepared for

the U.S. Department of Energy

under Contract DE-AC05-76RL01830

Pacific Northwest National Laboratory

Richland, Washington 99352 



\section{Executive Summary}

The Ultra-High Rate Germanium project at Pacific Northwest National Laboratory is conducting research to develop a high-purity germanium detector that can provide both the high resolution typical of germanium and high signal throughput. This report describes some of the initial radiation transport modeling efforts that have been conducted, such as the hit rate and power deposition per centimeter of germanium for a $1-\mathrm{MHz}{ }^{137} \mathrm{Cs}$ photon striking the front of the active area of the detector. In addition, this report describes the process used to generate the source spectrum for the spent fuel application evaluation. 



\section{Contents}

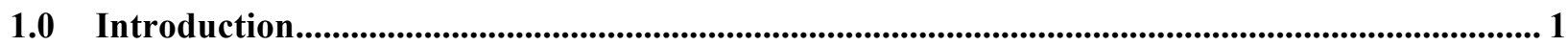

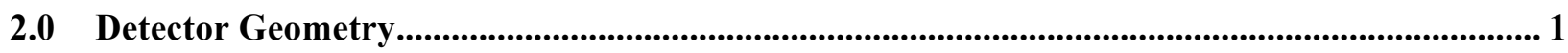

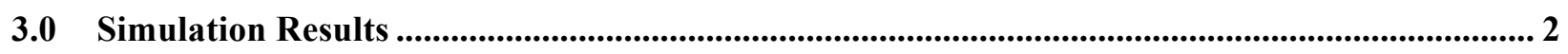

4.0 Source Modeling .......................................................................................................................................... 5

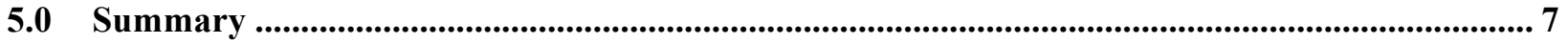

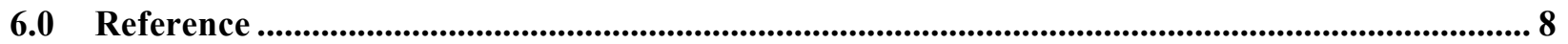





\subsection{Introduction}

The Ultra-High Rate Germanium (UHRGe) project at Pacific Northwest National Laboratory (PNNL) is conducting research to develop a high-purity germanium (HPGe) detector that can provide both the high resolution typical of germanium and high signal throughput. Such detectors may be beneficial for a variety of potential applications ranging from safeguards measurements of spent nuclear fuel to material detection and verification using active interrogation techniques. This report describes some of the initial radiation transport modeling efforts that have been conducted to help guide the design of the detector, as well as the process used to generate the source spectrum for the spent fuel application evaluation.

\subsection{Detector Geometry}

The detector was modeled using the radiation transport modeling framework of GEANT4 (Agostinelli et al., 2003). A simple detector layout was used to evaluate the initial design questions. This layout consists of 20 stacked layers of germanium disks $8 \mathrm{~cm}$ in diameter, as shown in Figure 1. There is $1 \mathrm{~mm}$ between each crystal. The front and side surfaces include a $0.6-\mathrm{mm}$ dead layer of germanium, which is typical for p-type germanium detectors. For some applications, n-type crystals may be more appropriate, in which case the location of the dead layer would change. A 1-mm-thick wall of aluminum surrounds the germanium crystals. The GEANT4 model records energy deposited in the layer; we have not included electric-field modeling for the charge transport in the germanium.

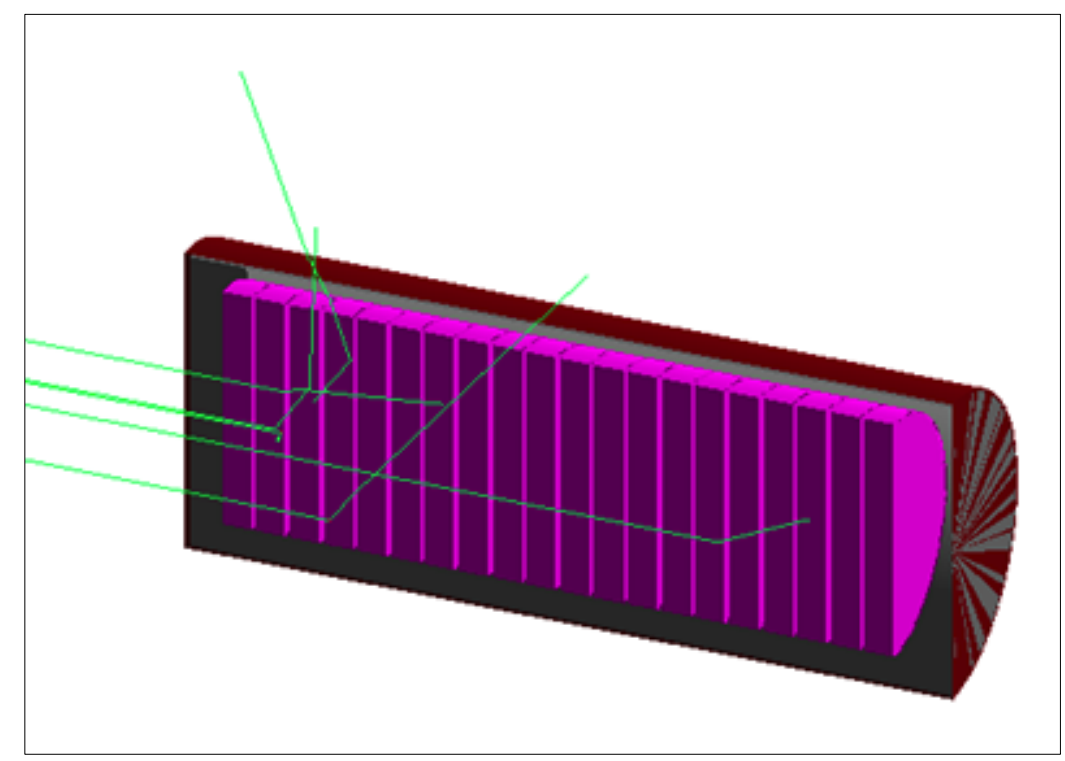

Figure 1: Cut-away diagram showing the inside of the test geometry for the UHRGe detector. Several tracks from $662-\mathrm{keV}$ photons are also shown. See the text for a description of the detector.

For this initial evaluation, the source is assumed to be in the far field. As a result, all photon tracks are parallel to the axis of symmetry of the detector. The entire front surface of the wall of the detector was illuminated by the photons, so that it is possible for photons that do not initially pass through the crystals 
to scatter in the outer wall of the detector and then pass through crystals. The energies of the photons were varied depending on the scenario being studied.

\subsection{Simulation Results}

The response of the detector to photons is shown in Figure 2. For these histograms, photons with discrete energies from $100 \mathrm{keV}$ to $6000 \mathrm{keV}$ are thrown at the detector. Some of those photons will pass through the detector without depositing any energy, some will deposit energy in a single layer, and some will deposit over multiple layers. It is important to note that these graphs are scored for any fraction of the initial photon energy deposited in the detector; it is not necessary for the photon to deposit all of its energy in the detector for it to be scored in these histograms. No threshold is applied to either the sum of energy deposited from the layers or the energy deposited in an individual layer in this initial modeling assessment.

The top left panel of Figure 2 shows the distribution of the multiplicity of detector of layers in which energy is deposited for several incident photon energies. The multiplicity distribution peaks at a single layer hit for all incident energies, but the distribution is considerably narrower for $100-\mathrm{keV}$ incident energy photons than for higher energies. Another issue relevant to the design of the detector is the distribution in layer number for the last layer to hit, which is shown in the upper right. This distribution peaks in the first layer (layer number 0) for all incident energies. Again, the distribution for $100-\mathrm{keV}$ photons is considerably narrower than the other distributions. The lower left graph shows the distribution for the number of layers between the first and last layer hit for a single photon. Again, the $100-\mathrm{keV}$ photon distribution is noticeably different from the others. The lower right graph is a re-expression of the upper right graph. In this graph, the cumulative probability is shown as a function of the last layer hit. This graph indicates, for example, that if one wants to observe some energy deposition from $1.5-\mathrm{MeV}$ photons in the detector $90 \%$ of the time, then one needs 11 layers, or $11 \mathrm{~cm}$ of germanium. The $80 \%$ efficiency for the $100-\mathrm{keV}$ photons is due to the 1-mm aluminum wall in front of the detector. 

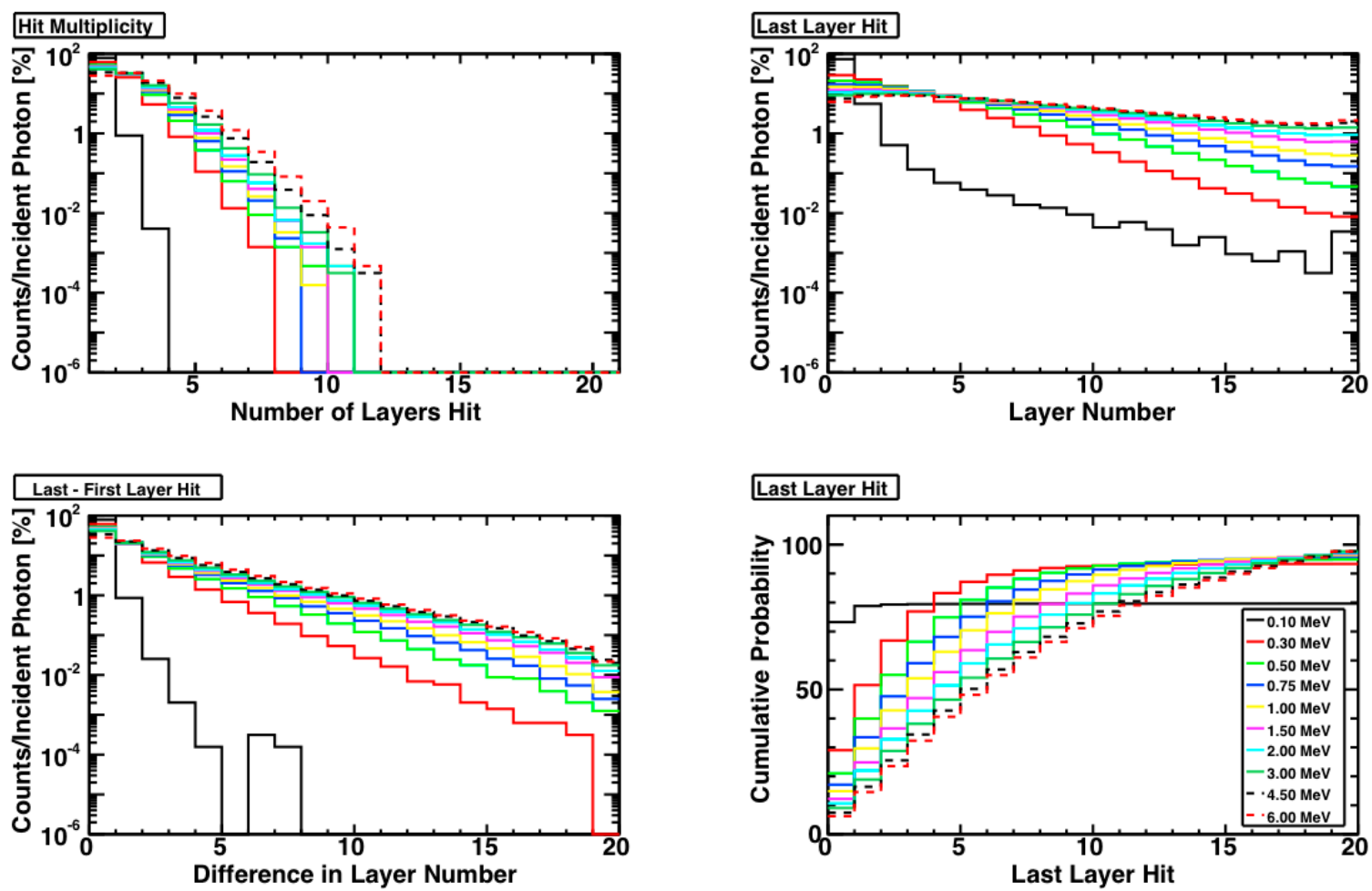

Figure 2: Histograms showing hit information in the detector for various photon energies. See the text for a description.

Figure 3 shows several plots that display both total and peak efficiencies as a function of incident photon energy and detector layer. The left column of this figure is related to total efficiency of the detector. The middle column is related to the peak efficiency, assuming only one layer is involved ("no add back"). The right column is related to peak efficiency, allowing for multiple layers to be combined to sum to the peak ("with add back"). For this column, the information is scored in the last layer hit. The top row shows the efficiency. The middle row shows the cumulative efficiency. The bottom row shows the cumulative efficiency scaled to 1 for the $20^{\text {th }}$ layer. The upper left graph is a repeat of the upper right graph in Figure 2. The plots showing the peak efficiencies with and without add-back show the importance of being able to use multiple layers to determine the peak energies. For instance, the maximum peak efficiency for a $3-\mathrm{MeV}$ incident photon is roughly $3 \%$ without add-back, but $14 \%$ with add-back, as seen in the middle row and middle and right column plots. The relative cumulate peak efficiency plots are helpful in determining the advantage of a thicker detector. For instance, with addback one can collect $80 \%$ of the peak efficiency for 11 layers $(11 \mathrm{~cm})$ of the detector, compared to all 20 layers $(20 \mathrm{~cm})$. The relative cumulate peak efficiencies without add-back converge more rapidly because the prior layers serve simply as attenuation for the later layers. Note that the peak efficiencies account only for depositions of the total energy of the photon, not for the first or second escape peaks, so that peak efficiencies for the highest energy photons may be underestimated relative to how they would be implemented in practice.

One application envisioned for the UHRGe detector is the assay of spent nuclear fuel. The $662-\mathrm{keV}$ line from ${ }^{137} \mathrm{Cs}$ heavily dominates the spent fuel spectra. To begin to understand the detector requirements for 
this application, the detector was modeled using $662-\mathrm{keV}$ photons incident on the detector. The results, normalized to $1 \mathrm{MHz}$ of $662-\mathrm{keV}$ photons incident on the active area of the detector (1-MHz photons over the 8-cm-diameter crystal face), are shown in Figure 4. The energy deposited per second is shown in the upper left plot. The distribution peaks in the first layer at $100 \mathrm{GeV} / \mathrm{s}$. The upper right plot shows the average energy deposited for photons hitting that layer. This quantity is determined by calculating the total energy deposited in that layer by the number of hits in that layer. Note that the zero is suppressed in this plot, so that the average energy deposition is relatively flat. The lower left plot shows the count rate as a function of layer number (Detector Depth). Roughly one-third of the 662-keV photons will deposit some energy in the first layer. The count rate is reduced by one-half by the fifth layer.
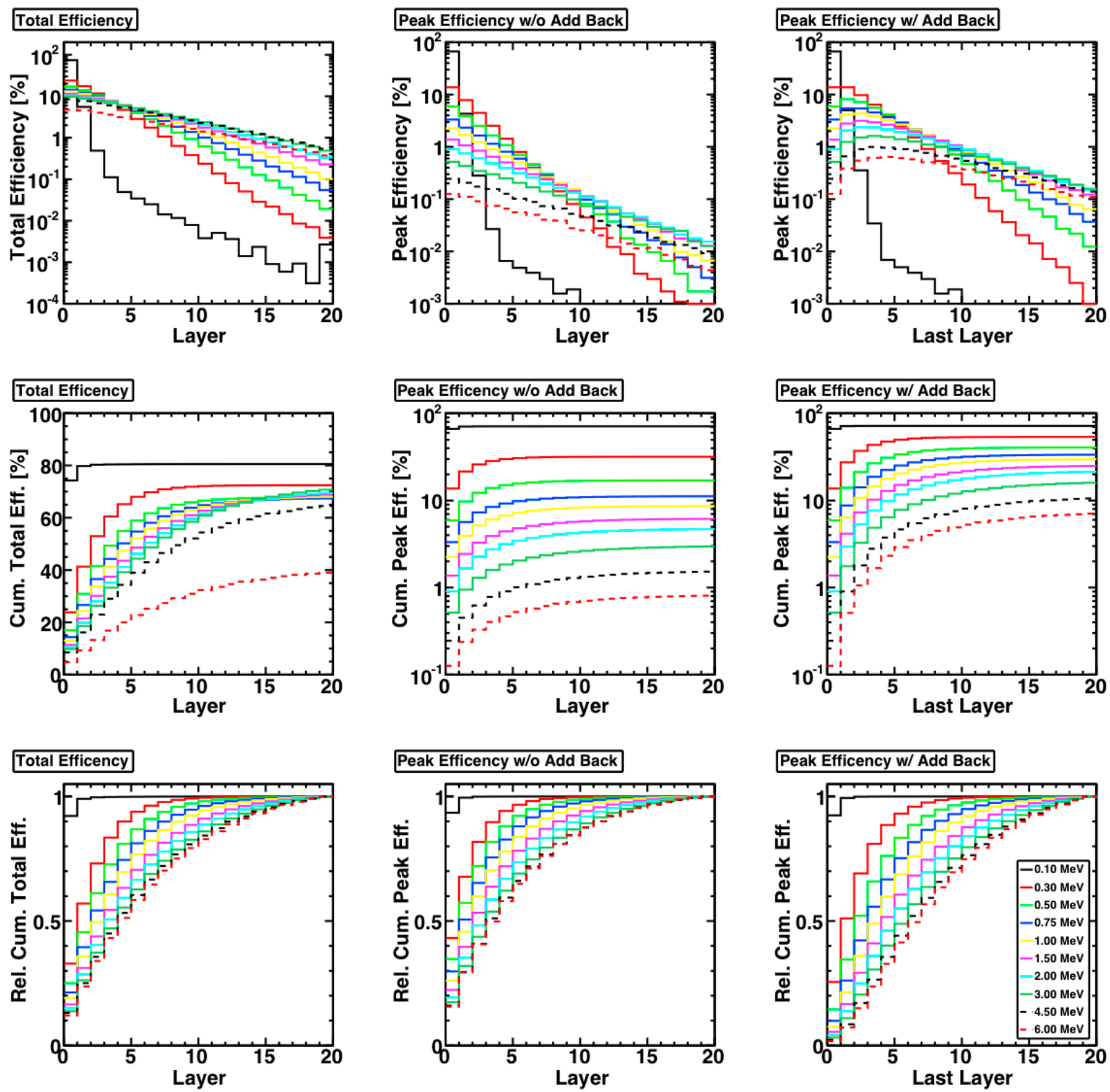

Figure 3: Efficiency histograms for a variety of incident photon energies. See text for description. 

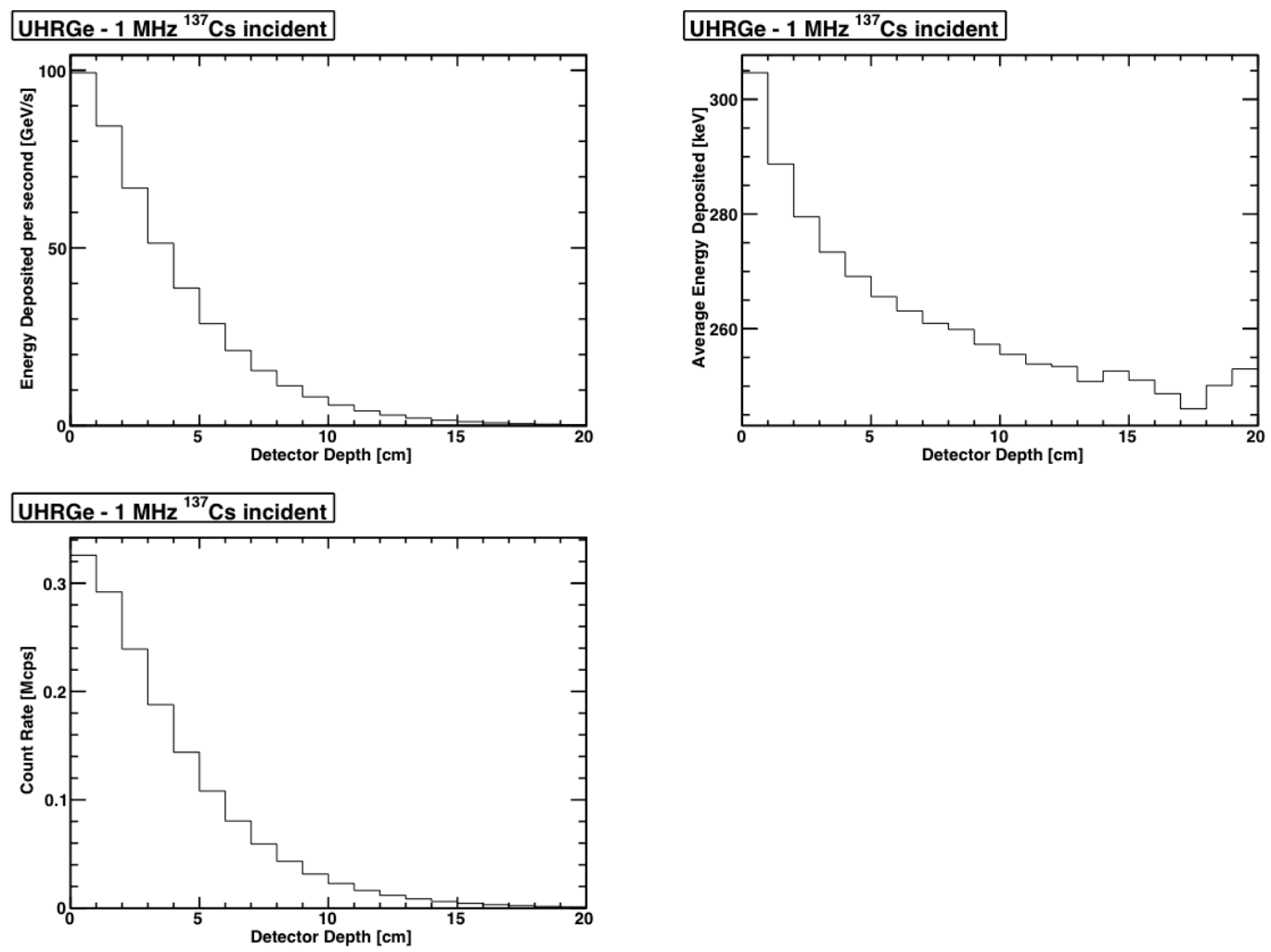

Figure 4: Hit information for $1 \mathrm{MHz}$ of $662-\mathrm{keV}$ photons incident on the active area of the detector

\subsection{Source Modeling}

One of the potential applications of the UHRGe detector is the assay of spent nuclear fuel. The intense gamma-ray field emitted by the spent fuel is a challenge for most detectors. One specific approach of interest is high-energy delayed gammas induced by active interrogation of the fuel with a neutron source. Above $3 \mathrm{MeV}$, there is very little passive radiation from the spent fuel, so that the delay-gamma signatures have negligible backgrounds to compete against. To facilitate the evaluation of UHRGe for such an application, we are currently developing a model for gamma-ray spectra for this application, which includes both the passive background from the fuel and the actively induced signal of the delayed gammas.

There are several steps involved in generating the passive background gamma-ray spectra from spent fuel. The initial step is a determination of the various isotopic masses in the spent fuel. We extract the masses from the first version of the Next Generation Safeguards Initiative (NGSI) spent fuel library (NGSI 64), which consists of sixty-four 17x17 assemblies that vary in initial enrichment, burnup and cooling time (Tobin et al., 2009). The baseline assembly used by the delayed-gamma effort has 30-giga-Watt-days per 
metric ton of uranium burn-up, $3 \%$ initial enrichment, and a 20 -year cooling time. The program SYNTH (Hensley et al., 1995) is then used to determine the number of gamma rays and X-rays generated from each of the isotopes. The production rates of this assembly are shown in Figure 5. These production rates are then used as the energy-probability input source in MCNP. The photons are attenuated by the fuel, and those escaping the assembly are tallied as surface emissions for further studies.

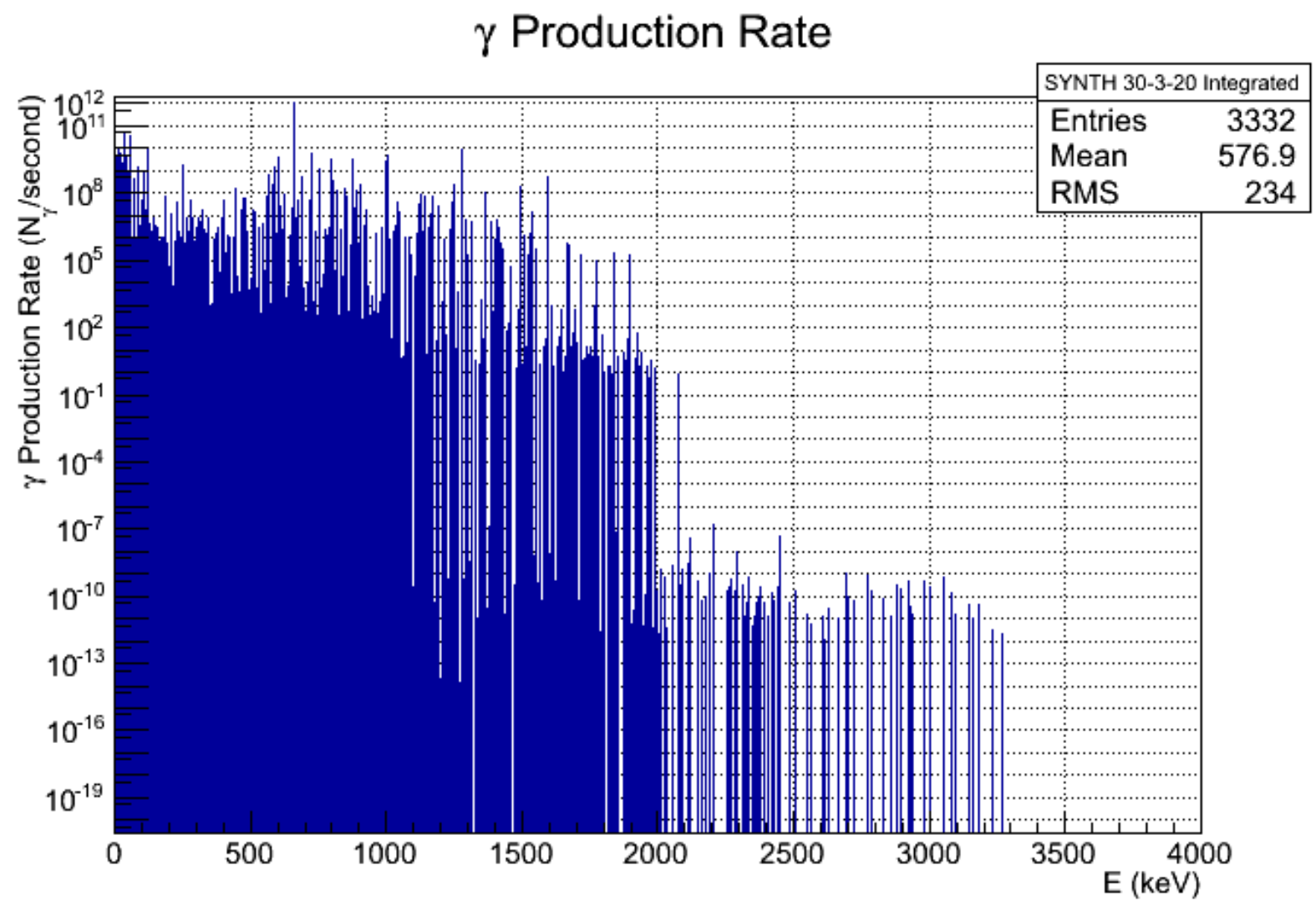

Figure 5: The photon production rates of the integrated masses of the NGSI spent fuel assembly model with $30 \mathrm{GWD} / \mathrm{gU}$ burn-up, 3\% initial enrichment, and a 20 -year cooling time. Emission of these lines is dependent upon self-shielding attenuation.

The same NGSI spent fuel assemblies are used to simulate the active-induced signal of the delayed gammas. The delayed-gamma simulations are performed by irradiating the assembly with a 14-MeV deuterium-tritium neutron source (Campbell et al, 2011). The dominant passive gamma line is $662 \mathrm{keV}$, and there are 4-8 orders of magnitude between this line and those in the 3-6 MeV range (see Figure 5). To validate these passive radiation simulations, measurements of actual spent fuel samples are being performed and analyzed. 


\subsection{Summary}

Initial radiation transport modeling results have been presented for a conceptual UHRGe detector. The objective of this initial study is to begin to understand the performance of the detector in a high-rate gamma-ray environment. A conceptual model of 20 layers of 8-cm-radius,1-cm-thick, high-purity germanium disks was developed. The performance of these systems for a variety of photon fields was explored. For instance, it was shown that $11 \mathrm{~cm}$ of germanium will provide $80 \%$ of the peak counts for $20 \mathrm{~cm}$ of germanium for photons below $3 \mathrm{MeV}$. This information will be useful in determining the depth of the UHRGe detector. This report also discussed the process for how spectra are generated for the application of measuring delayed gamma-rays from a spent nuclear fuel assembly that has been exposed to a high flux of neutrons. The tools discussed in this paper will be used in the design of the UHRGe detector. 


\subsection{Reference}

Agostinelli, S, et al., 2003. "Geant4 - a Simulation Toolkit." Nucl. Instrum. Methods Phys. Res. A, Accel. Spectrom. Detect. Assoc. Equip. (Netherlands) 506(3):250-303.

Campbell, LW, LE Smith, and AC Misner. 2011. "High-Energy Delayed Gamma Spectroscopy for Spent Nuclear Fuel Assay." IEEE Transactions on Nuclear Science 58(Copyright 2011, The Institution of Engineering and Technology):231-40.

Hensley, WK, AD McKinnon, HS Miley, ME Panisko, and RM Savard. 1995. Synth - a Spectrum Synthesizer. In Proceedings 229-37 pp.

Tobin, SJ, MA Schear, A Rajasingum, NP Sandoval, BA Ludewigt, BJ Quiter, ML Fensin, M T Swinhoe, SJ Thompson, SY Lee, HO Menlove, and LE Smith. 2009. "Determination of Plutonium Content in Spent Fuel with Nondestructive Assay," in Proceedings of the $50^{\text {th }}$ Annual Meeting of the Institute of Nuclear Materials Management, July 12-15, 2009, Tucson, AZ. Institute of Nuclear Materials Management, Northbrook, Illinois. 



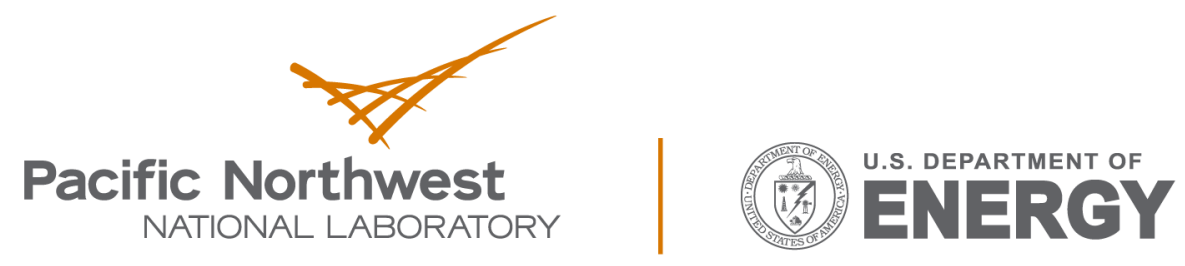

Proudly Operated by Battelle Since 1965

902 Battelle Boulevard

P.O. Box 999

Richland, WA 99352

1-888-375-PNNL (7665)

www.pnnl.gov 